\title{
Commentary on Sood et al.: "Cochrane Systematic Reviews in Acupuncture: Methodological Diversity in Database Searching"
}

\author{
LIXING LAO, Ph.D., L.Ac.
}

B ecause systematic reviews are the foundation of evidence-based medicine, the number of systematic reviews has been growing rapidly in recent years, and Cochrane reviews remain the acknowledged gold standard of high quality. Sood et al. reported their interesting finding on the range of databases searched in Cochrane acupuncture reviews (see page 719-722 in this issue). They hypothesize that the lack of a standardized search method across reviews may be producing bias.

In an age of limited time and resources, it is important to know how to leverage resources without compromising validity. There are hundreds of databases. A helpful next step would be to test this more-is-better hypothesis to see whether searching more databases actually yields more relevant trials. One might start, for example, with the three highest yielding databases-MEDLINE ${ }^{\circledR}$, Embase, and the Cochrane Controlled Trials Registry. One would document the yield of relevant trials from those databases and then search the other databases to see whether the additional database search yields additional trials, and if so, whether these additional trials actually change the results of the review.

Sood et al. state that failing to include non-English language studies may introduce bias, citing the Moher et al. ${ }^{1}$ analysis of reviews of complementary and alternative medicine (CAM). Unfortunately that sample lumps all CAM modalities together. It is known that there can be language bias in herbal reviews, ${ }^{2}$ and several herbal reviews are included in the sample. However, it is not known whether such a language bias exists for acupuncture reviews, and the Moher et al. study contained only two acupuncture reviews, , $^{3,4}$ a sample too small to provide meaningful conclusions.

As a Chinese practitioner who received acupuncture training in China and as a researcher who received training in the West, I find the discussion about Chinese language trials particularly of interest. There have been citations in the literature suggesting that Chinese trials are of a low methodological quality. Ter Reit et al. ${ }^{5}$ have noted the poor qual- ity of Chinese acupuncture trials. Vickers et al. ${ }^{6}$ showed that $100 \%$ of the acupuncture trials published from China showed positive results, a figure suggesting publication bias or poor methodological quality or both. Liu et al. ${ }^{7}$ showed randomization methods in Chinese randomized controlled trials (RCTs) are usually inadequate. This translates into the pragmatic question as to whether it is worth the time and expense to search the Chinese literature.

One solution for enhancing the language comprehensiveness of Cochrane reviews is to establish more Cochrane centers across the world and to enhance collaboration among Cochrane centers in these different countries. The most costand time-efficient way to search a foreign language database is to collaborate with native (or local) researchers who speak that language.

When I am invited back to China to present our research data of randomized trials, I am often asked to provide further workshops on research methodology including methods of randomization and adequate control issues - an important and positive sign that the interest in conducting high-quality randomized trials is growing in China. Along with the increased awareness of evidence-based medicine and the importance of conducting scientifically sound RCTs in developing countries such as China, we would expect more high quality RCTs to be published. Therefore, whatever the outcome of including or not including foreign language trials in acupuncture reviews, these findings should be re-evaluated periodically, for we live in an international scientific community where things are undergoing very rapid change.

\section{REFERENCES}

1. Moher D, Pham B, Lawson ML, Klassen TP. The inclusion of reports of randomized trials published in languages other than English in systematic reviews. Health Technol Assess 2003; 7:1-90.

Center for Integrative Medicine, University of Maryland, Baltimore, MD. 
2. Ezzo J, Berman B, Vickers A, Linde K. Complementary medicine and the Cochrane Collaboration. JAMA 1998;280: 1628-1630.

3. White AR, Rampes H, Ernst E. Acupuncture for smoking cessation. Cochrane Database Syst Rev 2000;350:CD000009.

4. Lee A, Done ML. The use of nonpharmacologic techniques to prevent postoperative nausea and vomiting: A meta-analysis. Anesth Analg 1999;88:1362-1369.

5. Ter Reit GK, Kleijnan J, Knipschild P. Acupuncture and chronic pain: A criteria-based meta-analysis. J Clin Epidemiol 1990;43: 1191-2000.

6. Vickers A, Harland R, Goyal N, Rees R. Do certain countries produce only positive results? Sixth International Cochrane Colloquium, Baltimore: October 22-26, 1998, Abstract A18.
7. Liu J, Kjaergard LL, Gluud C. Misuse of randomization: A review of Chinese randomized trials of herbal medicines for chronic hepatitis B. Am J Chin Med 2002;30:173-176.

Address reprint requests to: Lixing Lao, Ph.D., L.Ac. Center For Integrative Medicine University of Maryland Kernan Hospital Mansion, 2200 Kernan Drive Baltimore, MD, 21207

E-mail: LLao@compmed.umm.edu 